\title{
Linear and nonlinear causality in the UK housing market: a regional approach
}

\author{
Eleni Kyriazakou • Theodore Panagiotidis* \\ Department of Economics, University of Macedonia, Greece
}

Received: 7 October 2014

Revised: 12 December 2014

Accepted: 3 January 2015

\begin{abstract}
We examine the causal relationship between 12 UK regional house prices. Our data span from 1983:Q1 to 2012:Q4. The causal linkages both for the first differences and the levels are examined via Granger causality. The former allows us to examine short-run predictability while the latter the long-run. We relax the assumption of linearity and examine nonlinear pair-wise causality following Diks and Panchenko (2006) both for the levels and the first differences. We find that long-run causality among the regions is mainly linear while in the short-run is nonlinear. London's effect on the other regions is found to be mainly nonlinear in the short-run.
\end{abstract}

Keywords: house prices, regions, linear and nonlinear Granger causality

JEL Classification Codes: R11, R31

\section{Introduction}

The housing market had always a dominant role in the economy. It is the single most important asset of every household. The interest for the behavior of UK regional house prices has increased over the past two decades. This is primarily due to the significant impact that house prices have on economic activity. The fluctuations in regional house prices have led to the examination of the interaction among them. This letter focuses on the predictability aspect (causality) amongst the UK regional housing market. We investigate whether we can identify the regions that drive (cause or predict) the rest of the UK regional housing market. We examine the linear causality among all the regions by employing a VAR (Vector Autoregressive) framework. Later, we relax the assumption of linearity with the nonparametric approach proposed more recently by Diks and Panchenko (2006) (DP hence forth).

\footnotetext{
*Corresponding author. E-mail: tpanag@uom.gr.

Citation: Kyriazakou, E. and T. Panagiotidis (2014) Linear and nonlinear causality in the UK housing market: a regional approach, Economics and Business Letters, 3(4), 288-297.
} 
Starting with the work of Meen (1999), it has been argued that shocks to regional house prices ripple out across the economy and concluded that there is a long run equilibrium relationship between regional house prices in the UK. Tsai (2014) re-examined the ripple effect for the UK. Panel unit root tests indicate that the relative price and volume ratios point towards stationarity signifying a long-run relationship between regional and national housing in the UK. According to Cook (2003), the form of asymmetry detected varies between regions of the UK. Regions in the South East experience faster convergence following downswings in prices while other regions exhibit more rapid convergence following the increases in prices. Pesaran (2009) supports that the effects of a shock decay more slowly along the geographical dimension as compared to the decay along the time dimension. Moreover, Holmes and Grimes (2008) employ co-integration techniques and argue that all UK regional house prices are driven by a single common stochastic trend and that London exhibits the highest degree of persistence. Finally, Holmes et al (2011), using a pair-wise approach and unit root tests, is investigating regional house price convergence in the US. They find that convergence is stronger between contiguous than non-contiguous regions. Kuethe and Pede (2011) incorporate locational spillovers through a spatial econometric adaptation of vector autoregression (SpVAR). The results suggest these spillovers may Granger cause housing price movements in a large number of cases.

In this paper we investigate linear and nonlinear (in the DP sense) convergence for $12 \mathrm{UK}$ regions for a period that spans from 1983:1 till 2012:4 (120 observations). In contrast to the literature, we allow nonlinear dynamics to drive convergence.

The rest of the paper is organized as follows: Section 2 briefly discusses the methodology, section 3 presents the data, the results appear in section 4 and the last section concludes.

\section{Methods}

The econometric methodology employed in this strand of the literature is derived within the linear framework proposed initially by Granger (1969) and later by Sims (1980). The latter is usually referred to as the short-run measure of causality (predictability) as we typically employ first differences. Toda and Yamamoto (TY) (1995) extended the analysis in the long-run allowing for the possibility of co-integration. They recommend adding an extra lag in the analysis of the levels of the variables.

We will start our analysis by adopting a VAR (Vector Autoregressive) framework:

$$
\Delta y_{\mathrm{t}}=\beta_{0}+\beta_{1} \Delta y_{\mathrm{t}-1}+\beta_{2} \Delta y_{\mathrm{t}-2}+\ldots+u_{t}
$$

where $\Delta y_{t}$ is a vector with dimension $(12 \times 1)$ and the lag length is based on the SIC (Schwartz Information Criterion). Linear Granger causality is based on the $F$-tests (see Brooks 2014 pages 333-335 for more details).The analysis above assumes linearity and is short run.

In the long-run we follow TY and employ a VAR framework similar to the above but for the levels of the series $\left(y_{t}\right)$. The lag length is based on the SIC but in the TY we need to add one lag. Hiemstra and Jones (1994) proposed a test that can detect nonlinear causality. DP extended the latter by proposing a nonparametric approach. In this analysis, we are employing DP to allow for nonlinear causality between the UK regional housing markets. For more discussion on the tests and applications, the reader is pointed to Alagidede et al. (2011) and Bampinas and Panagiotidis (2014). 


\section{Data}

The data we employ are quarterly observations of the natural logarithm of regional house prices and the UK (as a whole) over the period 1983Q1-2012Q4 (120 observations totally). The dataset under consideration were collected from the Halifax House Price Index (available at www.lloydsbankinggroup.com).

Our regional analysis includes the following regions:

- Scotland

- Wales

- Northern Ireland

- London

- South West

- South East

- East Anglia

- East Midlands

- West Midlands

- North East

- North West

- Yorkshire

Figure 1 plots the level of the log series (employed for the long-run analysis) whereas Figure 2 plots the first differences (growth rates) (for the short analysis).

Figure 1. Log levels

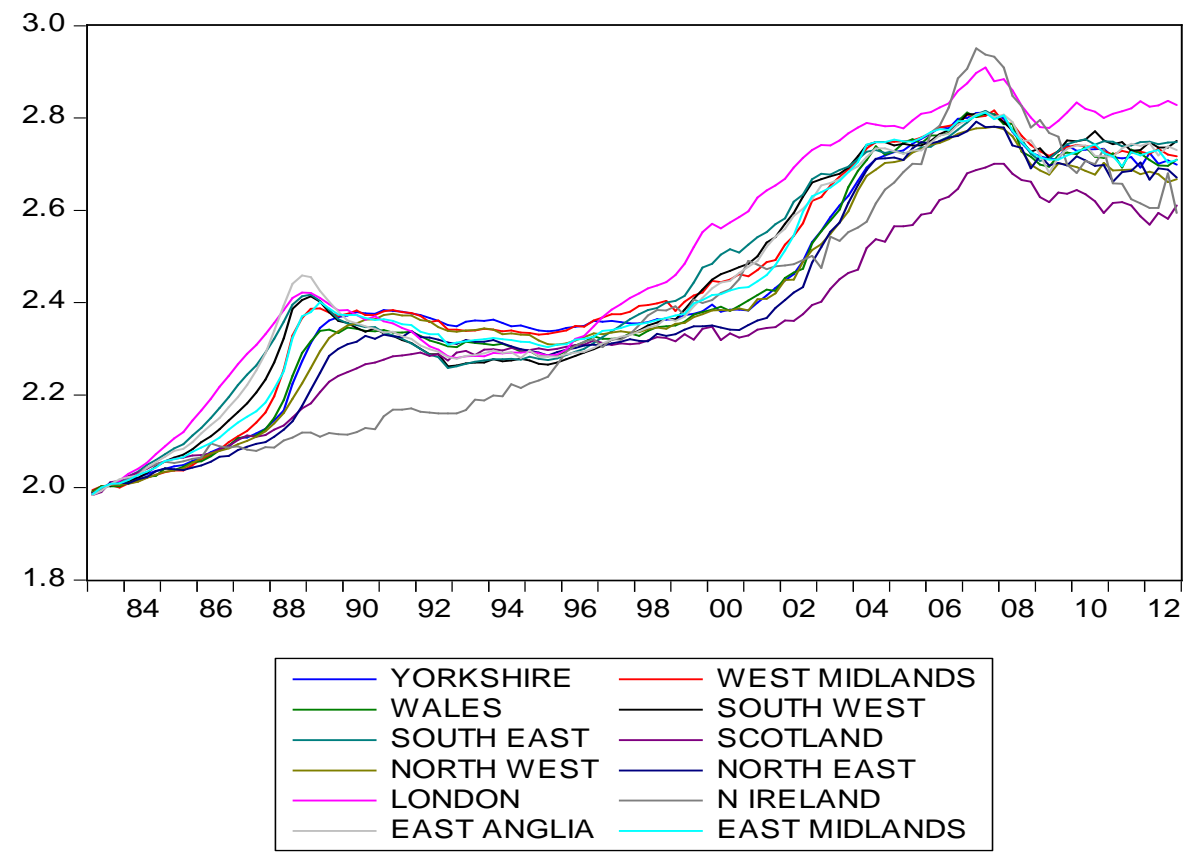


Figure 2. First differences

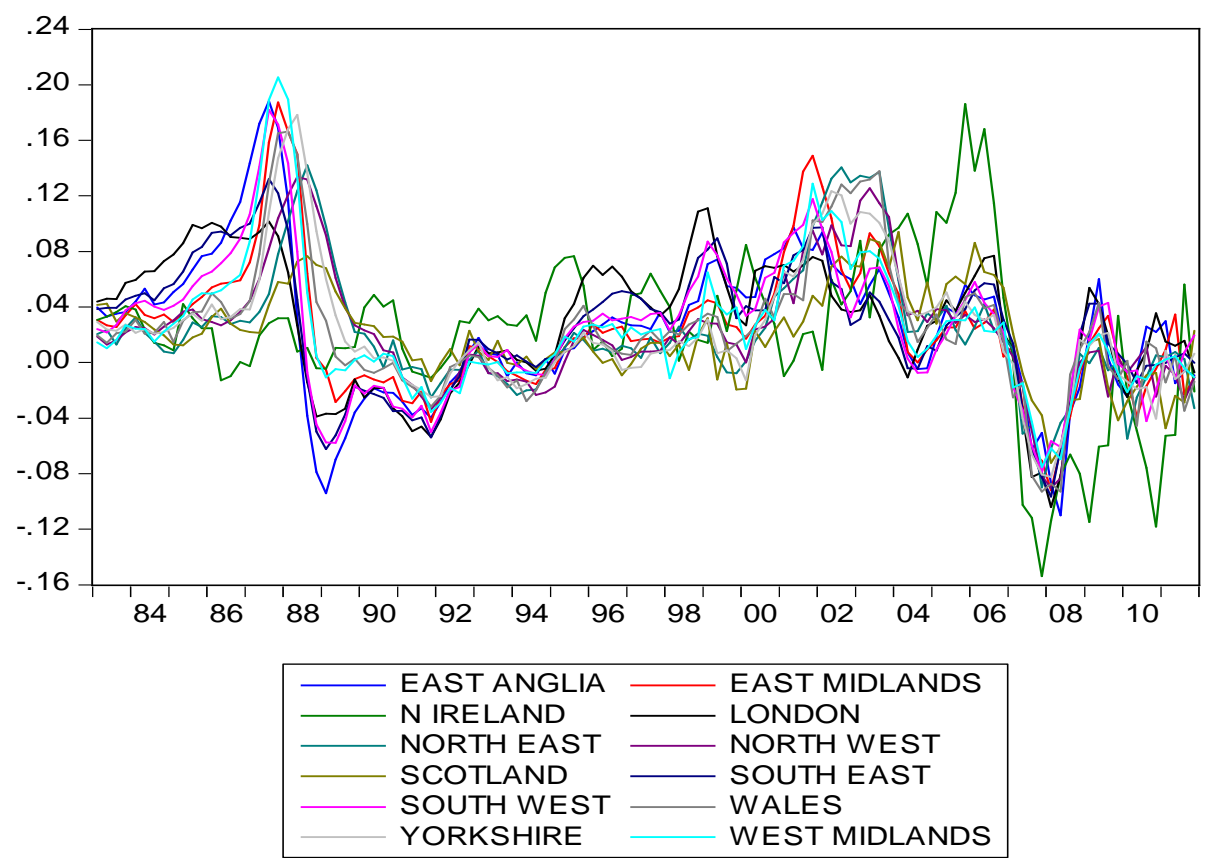

\section{Results}

Our analysis starts from a linear VAR specification. The lag-length is chosen using the SIC (lag length equals to 1 for the short run and 2 for the long run; see notes in Tables 1 to 4). Standard linear Granger causality is then performed using a VAR specification that contains first differences of all 12 UK regions. We refer to this as the short-run analysis. The results for this are presented in Table 1.We can argue that the South East, East Midlands and Scotland have the highest significant results ( 5 out of the 11 pairs). In other words, these are the regions that drive the short-run (first differences) linear predictability.

The analysis mentioned above assumed that there is no long-run relationship between the variables. To allow for co-integration we adopt the TY approach. As a result the next step would be to construct a VAR with the levels $\left(y_{t}\right)$ of all $12 \mathrm{UK}$ regional housing market indices. We include one more lag (SIC plus 1) following Toda and Yamamoto (1995). We then perform a standard linear $F$-test. The results are summarized in Table 2 . We refer to these as the linear long-run causality. The regions with the more significant effect on the others are: the South West and West Midlands (10 out of the 11 pairs and 9 out of 11 significant $p$-values accordingly). In the long-run we do get more significant pairs than in the short-run. The latter suggests that there is more linear predictability in the long-run rather than in the sort-run.

Given that there is no reason to assume that the convergence process is a linear one, we turn to the nonlinear analysis. This is a pair-wise analysis as we consider the interaction between all the possible pairs (12 UK regions plus the UK aggregate Index). Table 3 presents the short-run (first differences) results of the test statistic proposed by Diks and Panchenko (2006). The highest portion of significant $p$-values is observed in the case of Wales (11 out of the 12 pairs) followed by London (10 out of 12 pairs), then UK (this is the aggregate index, 9 out of 12 pairs) and the North West (9 out of 12). London's predictability is mainly a short-run and nonlinear phenomena and its effect is more important than the overall UK aggregate index. 
Table 1. Linear causality (short-run first differences)

\begin{tabular}{|c|c|c|c|c|c|c|c|c|c|c|c|c|}
\hline NDONA & $>$ lond & $>$ north & $>$ york & $>$ swest & $>$ seast & > eanglia & >emidl & $>$ nwest & $>$ wmidl & $>$ wales & $>s \cot$ & >irel \\
\hline$p$-values & & 0.3104 & 0.8796 & 0.1108 & 0.6866 & 0.9662 & 0.0508 & 0.4535 & $0.0725^{*}$ & 0.2612 & $0.0121^{* *}$ & $0.0445^{* *}$ \\
\hline NORTHA & >lond & $>$ north & $>$ york & > swest & $>$ seast & > eanglia & >emidl & $>$ nwest & wmidl & $>$ wales & $>s \cot$ & >irel \\
\hline$p$-values & 0.1182 & & 0.1118 & 0.6025 & $0.0422^{* *}$ & 0.8560 & 0.4516 & 0.2917 & 0.9607 & $0.0014^{* * *}$ & 0.2961 & 0.3818 \\
\hline YORKA & $>$ lond & $>$ north & $>$ york & > swest & > seast & > eanglia & > emidl & $>$ nwest & $>$ wmidl & $>$ wales & $>s \cot$ & >irel \\
\hline p-values & 0.9011 & $0.0177^{* *}$ & & $0.0748^{*}$ & 0.2159 & 0.4603 & 0.7961 & 0.1191 & 0.3347 & $0.0611^{*}$ & 0.2356 & 0.4793 \\
\hline SOUTH WE & $>$ lond & $>$ north & $>$ york & > swest & $>$ seast & > eanglia & >emidl & $>$ nwest & wmidl & $>$ wales & $>s \cot$ & >irel \\
\hline$p$-values & 0.1804 & $0.0112^{* *}$ & $0.0461^{* *}$ & & 0.4749 & $0.0023^{* * *}$ & 0.9556 & 0.9355 & 0.1728 & 0.7562 & 0.4239 & 0.9619 \\
\hline SOUTHEA & $>$ lond & $>$ north & $>$ york & > swest & $>$ seast & > eanglia & >emidl & $>$ nwest & $>$ wmidl & $>$ wales & $>s c o t$ & >irel \\
\hline$p$-values & $0002^{*}$ & $0.0206^{* *}$ & 0.2176 & $0.0002^{* * *}$ & & 0.6193 & $0.0802^{*}$ & 0.6041 & 0.3317 & 0.6926 & $0.053^{*}$ & 0.2482 \\
\hline EAST & $>$ lond & $>$ north & $>$ york & > swest & $>$ seast & > eanglia & >emidl & >nwest & $>$ wmidl & $>$ wales & $>s \cot$ & >irel \\
\hline p-values & 0.5797 & $0.0066^{* * *}$ & $0.0538^{*}$ & $0.0688^{*}$ & 0.2205 & & $0.0147^{* *}$ & 0.3072 & 0.9576 & 0.1490 & 0.6892 & 0.5415 \\
\hline EAST MIDLA & $>$ lond & $>$ north & $>$ york & swest & $>$ seast & > eanglia & >emidl & >nwest & $>$ wmidl & $>$ wales & $>s \cot$ & >irel \\
\hline$p$-values & 0.3613 & $0.0018^{* * *}$ & 0.9094 & $0.0009^{* * *}$ & $0.0659 *$ & $0.0003^{* * *}$ & & 0.9031 & 0.6644 & 0.1443 & $0.0662^{*}$ & 0.7542 \\
\hline NORTH W & $>$ lond & $>$ north & $>$ york & > swest & $>$ seast & > eanglia & >emidl & $>$ nwest & $>$ wmidl & $>$ wales & $>s \cot$ & >irel \\
\hline$p$-values & 0.8123 & $0.0167^{* *}$ & $0.0096^{* * *}$ & $0.0271^{* *}$ & 0.7080 & 0.6392 & 0.2363 & & 0.6188 & 0.3408 & $0.0513^{*}$ & 0.4707 \\
\hline WEST MIDLANDA & $>$ lond & $>$ north & $>$ york & > swest & $>$ seast & > eanglia & >emidl & > nwest & $>$ wmidl & $>$ wales & $>s \cot$ & >irel \\
\hline p-values & 0.9761 & 0.1545 & 0.6433 & 0.4726 & 0.8075 & $0.0012^{* * *}$ & 0.8682 & 0.2238 & & 0.2501 & 0.1471 & 0.9455 \\
\hline WALESA & $>$ lond & $>$ north & $>$ york & $>$ swest & $>$ seast & > eanglia & >emidl & >nwest & $>$ wmidl & $>$ wales & $>s \cot$ & >irel \\
\hline$p$-values & 0.9986 & $0.0051^{* * *}$ & $0.0784^{*}$ & 0.5388 & 0.9481 & 0.1376 & 0.1571 & 0.2384 & 0.1109 & & $0.0419 * *$ & 0.1397 \\
\hline SCOTL & $>$ lond & $>$ north & $>$ york & > swest & $>$ seast & > eanglia & > emidl & $>$ nwest & $>$ wmidl & $>$ wales & $>s c o t$ & >irel \\
\hline$p$-values & $0.0526^{*}$ & 0.2503 & $0.0008^{* * *}$ & 0.7765 & $0.0795^{*}$ & 0.2779 & $0.0647^{*}$ & 0.1921 & 0.2299 & 0.6415 & & $0.0000^{* * *}$ \\
\hline IRELANDA & $>$ lond & $>$ north & $>$ york & > swest & $>$ seast & > eanglia & >emidl & $>$ nwest & $>$ wmidl & $>$ wales & $>s c 0 t$ & >irel \\
\hline$p$-values & $0.0523^{*}$ & 0.1446 & 0.2938 & 0.3139 & $0.0378^{* *}$ & 0.3855 & 0.1006 & $0.0101^{* *}$ & 0.5148 & 0.9810 & $0.0079^{* * *}$ & \\
\hline
\end{tabular}

Notes: $\rightarrow$ denotes the no-causality null hypothesis on first differences. The Schwarz Information Criterion was used to determine the optimal lag lengths for VAR $(p)$ models. The $p$-values with $* * *, * * *$ represent the significance at $10 \%$, $5 \%$ and $1 \%$ level respectively. Lag length equals 1 . 
Table 2. Linear causality (long-run, levels T\&Y)

\begin{tabular}{|c|c|c|c|c|c|c|c|c|c|c|c|c|}
\hline LONDONA & $>$ lond & $>$ north & $>$ york & > swest & $>$ seast & > eanglia & >emidl & >nwest & $>$ wmidl & $>$ wales & $>s c o t$ & >irel \\
\hline$p$-values & & 0.1478 & 0.3872 & 0.2583 & $0.0451^{* *}$ & 0.1444 & $0.0113^{* *}$ & 0.1712 & 0.8090 & $0.0115^{* *}$ & 0.3259 & $0.0403^{* *}$ \\
\hline NORTHA & $>$ lond & $>$ north & $>$ york & > swest & $>$ seast & > eanglia & >emidl & >nwest & $>$ wmidl & $>$ wales & $>s c o t$ & >irel \\
\hline$p$-values & 0.1281 & & $0.0084^{* * *}$ & $0.0037^{* * *}$ & $0.0003^{* * *}$ & 0.6888 & 0.9121 & $0.0442^{* *}$ & 0.1165 & $0.0001^{* * *}$ & $0.0001^{* * *}$ & $0.0004^{* * *}$ \\
\hline YORKA & $>$ lond & $>$ north & > york & > swest & $>$ seast & > eanglia & >emidl & >nwest & $>$ wmidl & $>$ wales & $>s \cot$ & >irel \\
\hline$p$-values & $0.0196 * *$ & 0.1605 & & $0.0000^{* * *}$ & $0.08300^{*}$ & $0.0004^{* * *}$ & $0.0687^{*}$ & $0.0032^{* * *}$ & $0.0000^{* * *}$ & 0.2180 & 0.1331 & 0.3638 \\
\hline SOUTH WESTA & $>$ lond & $>$ north & $>$ york & > swest & $>$ seast & > eanglia & >emidl & >nwest & $>$ wmidl & $>$ wales & $>s \cot$ & >irel \\
\hline$p$-values & $0.0041^{* * *}$ & $0.0000^{* * *}$ & $0.0245^{* *}$ & & $0.0537^{*}$ & $0.0000^{* * *}$ & $0.0301^{* *}$ & $0.0099 * * *$ & $0.0001^{* * *}$ & $0.0000^{* * *}$ & $0.0404^{* *}$ & 0.5033 \\
\hline SOUTH EASTA & $>$ lond & $>$ north & $>$ york & > swest & $>$ seast & > eanglia & > emidl & $>$ nwest & $>$ wmidl & $>$ wales & $>s \cot$ & >irel \\
\hline$p$-values & $0.0000^{* * *}$ & $0.0001^{* * *}$ & $0.0084^{* * *}$ & $0.0036^{* * *}$ & & $0.0321^{* *}$ & $0.0078^{* * *}$ & $0.0012^{* * *}$ & 0.3122 & $0.0000^{* * *}$ & 0.1576 & 0.1319 \\
\hline EAST ANGLIAA & $>$ lond & $>$ north & > york & > swest & $>$ seast & > eanglia & > emidl & >nwest & $>$ wmidl & $>$ wales & $>s c o t$ & >irel \\
\hline$p$-values & $0.0227^{* *}$ & $0.0000^{* * *}$ & 0.4197 & 0.9023 & $0.0002^{* * *}$ & & $0.0016^{* * *}$ & 0.0517 & $0.0421^{* *}$ & $0.0001^{* * *}$ & $0.0081^{* * *}$ & 0.4159 \\
\hline EAST MIDLANDA & $>$ lond & $>$ north & $>$ york & > swest & $>$ seast & > eanglia & > emidl & $>$ nwest & $>$ wmidl & $>$ wales & $>s \cot$ & >irel \\
\hline$p$-values & 0.3715 & $0.0000^{* * *}$ & 0.6046 & $0.0137^{* *}$ & $0.0547^{*}$ & $0.0000^{* * *}$ & & $0.0282^{* *}$ & $0.0000^{* * *}$ & $0.0003^{* * *}$ & $0.0367^{* *}$ & 0.5556 \\
\hline NORTH WESTA & $>$ lond & $>$ north & $>$ york & > swest & $>$ seast & > eanglia & >emidl & $>$ nwest & $>$ wmidl & $>$ wales & $>s \cot$ & >irel \\
\hline$p$-values & 0.1340 & $0.0770^{*}$ & $0.0082^{* * *}$ & $0.0094^{* * *}$ & 0.4057 & 0.1380 & 0.4688 & & $0.0043^{* * *}$ & 0.1289 & $0.0001^{* * *}$ & 0.3902 \\
\hline WEST MIDLANDA & $>$ lond & $>$ north & > york & > swest & $>$ seast & > eanglia & > emidl & >nwest & $>$ wmidl & $>$ wales & $>s \cot$ & >irel \\
\hline$p$-values & $0.0020 * * *$ & $0.0000^{* * *}$ & 0.6290 & $0.0333^{* *}$ & $0.0923^{*}$ & $0.0000^{* * *}$ & $0.0157^{* *}$ & $0.0151^{* *}$ & & $0.0000^{* * *}$ & $0.0188^{* *}$ & 0.6467 \\
\hline WALESA & $>$ lond & $>$ north & > york & > swest & $>$ seast & > eanglia & > emidl & >nwest & $>$ wmidl & $>$ wales & $>s \cot$ & >irel \\
\hline$p$-values & $0.0002^{* * *}$ & $0.0000^{* * *}$ & 0.1713 & $0.0860^{*}$ & 0.9534 & $0.0001^{* * *}$ & $0.0019^{* * *}$ & $0.0008^{* * *}$ & $0.0000^{* * *}$ & & $0.0100^{* *}$ & 0.3362 \\
\hline SCOTLANDA & $>$ lond & $>$ north & $>$ york & > swest & $>$ seast & > eanglia & > emidl & >nwest & $>$ wmidl & $>$ wales & $>s \cot$ & >irel \\
\hline$p$-values & 0.5413 & $0.007^{* * *}$ & $0.0028 * * *$ & 0.7501 & $0.0014^{* * *}$ & $0.0157^{* *}$ & $0.0014^{* * *}$ & $0.0241^{* *}$ & 0.1474 & $0.0354^{* *}$ & & $0.0000^{* * *}$ \\
\hline IRELANDA & $>$ lond & $>$ north & > york & > swest & $>$ seast & > eanglia & >emidl & >nwest & $>$ wmidl & $>$ wales & $>s \cot$ & >irel \\
\hline$p$-values & 0.5771 & 0.5122 & $0.0004^{* * *}$ & $0.0499 * *$ & $0.0622^{*}$ & 0.5294 & $0.0020^{* * *}$ & $0.0002^{* * *}$ & 0.3840 & 0.8240 & $0.0000^{* * *}$ & \\
\hline
\end{tabular}

Notes: $\nrightarrow$ denotes the no-causality null hypothesis on levels. The Schwarz Information Criterion was used to determine the optimal lag lengths for VAR $(p)$ models. The $p$-values with *,**,*** represent the significance at $10 \%, 5 \%$ and $1 \%$ level respectively. Lag length equals 2 (linear lag length +1 ). 
Table 3. Nonlinear (DP) causality (short-run, first differences)

\begin{tabular}{|c|c|c|c|c|c|c|c|c|c|c|c|c|c|}
\hline LONDON $\rightarrow$ & >lond & $>u k$ & >north & syork & >swest & >seast & >eanglia & >emidl & >nwest & $>$ wmidl & >wales & $>s \cot$ & >irel \\
\hline p-values & & $0.04242^{* *}$ & $0.06619^{*}$ & $0.08537^{*}$ & $0.00984^{* * *}$ & $0.01474^{* *}$ & $0.00522^{* * *}$ & $0.00639 * * *$ & $0.04937^{* *}$ & $0.00798 * * *$ & $0.03618^{* *}$ & 0.13877 & 0.39425 \\
\hline UKњ & >lond & >uk & >north & syork & >swest & seast & >eanglia & >emidl & >nwest & $>$ wmidl & >wales & $>s \cot$ & >irel \\
\hline p-values & 0.11415 & & $0.06532^{*}$ & 0.23257 & $0.02375^{* *}$ & $0.03445^{* *}$ & $0.05134^{*}$ & $0.01629 * *$ & $0.03671^{* *}$ & $0.00537^{* * *}$ & $0.00826 * * *$ & $0.09432^{*}$ & 0.28196 \\
\hline NORTHA & >lond & >uk & >north & >york & >swest & seast & >eanglia & >emidl & >nwest & $>$ wmidl & swales & $>s \cot$ & >irel \\
\hline p-values & $0.04844^{* *}$ & 0.13243 & & $0.06823^{*}$ & 0.17973 & 0.10458 & $0.04411^{* *}$ & $0.03703^{* *}$ & $0.02765^{* *}$ & 0.12443 & $0.00703^{* * *}$ & $0.06732^{*}$ & 0.26711 \\
\hline YORKA & >lond & >uk & >north & syork & >swest & sseast & >eanglia & >emidl & >nwest & >wmidl & >wales & $>s \cot$ & >irel \\
\hline$p$-values & 0.11217 & 0.33997 & $0.01254^{* *}$ & & 0.15830 & 0.10897 & $0.05544^{*}$ & $0.0495^{* *}$ & $0.04306^{* *}$ & 0.10309 & $0.01619^{* *}$ & $0.03404^{* *}$ & 0.65383 \\
\hline SOUTH WESTA & >lond & >uk & >north & >york & >swest & >seast & >eanglia & >emidl & >nwest & $>$ wmidl & >wales & $>s \cot$ & >irel \\
\hline$p$-values & $0.09239 *$ & 0.12730 & $0.02668^{* *}$ & 0.11247 & & $0.07488^{*}$ & $0.05151^{*}$ & $0.05461^{*}$ & $0.07166^{*}$ & 0.12426 & $0.03617^{* *}$ & $0.08254^{*}$ & 0.26532 \\
\hline SOUTHEASTA & >lond & >uk & >north & >york & >swest & >seast & >eanglia & >emidl & >nwest & >wmidl & swales & $>s \cot$ & >irel \\
\hline p-values & $0.04781^{* *}$ & $0.07071^{*}$ & 0.11294 & 0.15220 & $0.01362^{* *}$ & & $0.01874^{* *}$ & $0.01916^{* *}$ & $0.08740^{*}$ & $0.01872^{* *}$ & $0.08474^{*}$ & 0.10215 & 0.66267 \\
\hline EAST ANGLIAÆ & >lond & >uk & >north & syork & >swest & >seast & >eanglia & >emidl & >nwest & >wmidl & >wales & $>s \cot$ & >irel \\
\hline p-values & 0.12261 & 0.11909 & $0.04585^{* *}$ & $0.09868^{*}$ & $0.02856^{* *}$ & 0.10174 & & $0.04777^{* *}$ & $0.07554^{*}$ & $0.05870^{*}$ & $0.08064^{*}$ & $0.05668^{*}$ & 0.77420 \\
\hline EAST MIDLAND $\nrightarrow$ & >lond & >uk & >north & >york & >swest & >seast & >eanglia & >emidl & >nwest & $>$ wmidl & >wales & $>s \cot$ & >irel \\
\hline p-values & 0.35381 & 0.42557 & 0.11205 & 0.41095 & $0.03525^{* *}$ & 0.19082 & $0.04255^{* *}$ & & $0.07665^{*}$ & $0.02127^{* *}$ & $0.02638^{* *}$ & $0.09793^{*}$ & 0.32254 \\
\hline NORTH WESTA & >lond & >uk & >north & >york & >swest & >seast & >eanglia & >emidl & >nwest & >wmidl & swales & $>s \cot$ & sirel \\
\hline p-values & $0.02074^{* *}$ & $0.09426^{*}$ & $0.02219^{* *}$ & 0.41122 & 0.12180 & $0.06462^{*}$ & $0.09599 *$ & $0.04978^{* *}$ & & $0.05612^{*}$ & $0.0308^{* *}$ & $0.0321^{* *}$ & 0.78551 \\
\hline WEST MIDLANDA & >lond & >uk & >north & >york & >swest & >seast & >eanglia & semidl & >nwest & >wmidl & swales & $>s \cot$ & >irel \\
\hline p-values & 0.12647 & 0.13911 & $0.03803^{* *}$ & 0.27840 & 0.16174 & 0.13323 & $0.09688^{*}$ & $0.05277^{*}$ & 0.11815 & & $0.06509^{*}$ & 0.14640 & 0.28037 \\
\hline WALES $\rightarrow$ & $>$ lond & >uk & >north & >york & >swest & $>$ seast & >eanglia & >emidl & >nwest & $>$ wmidl & >wales & $>s \cot$ & >irel \\
\hline p-values & $0.0595^{*}$ & $0.07911^{*}$ & $0.00925^{* * *}$ & $0.02348^{* *}$ & $0.03001^{* *}$ & $0.08234^{*}$ & $0.06516^{*}$ & $0.02192^{* *}$ & $0.01252^{* *}$ & $0.02045^{* *}$ & & $0.08151^{*}$ & 0.19478 \\
\hline SCOTLANDA & >lond & >uk & >north & >york & >swest & >seast & >eanglia & semidl & >nwest & >wmidl & >wales & $>s \cot$ & >irel \\
\hline p-values & $0.04818^{* *}$ & 0.24635 & 0.10872 & 0.34166 & $0.07128 *$ & 0.41061 & $0.02214^{* *}$ & 0.15223 & 0.79159 & 0.76837 & $0.07844^{*}$ & & 0.34519 \\
\hline IRELANDA & >lond & >uk & >north & syork & >swest & seast & >eanglia & >emidl & >nwest & >wmidl & swales & $>s \cot$ & >irel \\
\hline p-values & $0.05441^{*}$ & 0.28341 & 0.28809 & $0.07923^{*}$ & 0.14701 & $0.07238^{*}$ & $0.05451^{*}$ & $0.07136^{*}$ & 0.12176 & 0.29254 & $0.08345^{*}$ & 0.13917 & \\
\hline
\end{tabular}

Notes: This table reports the results of nonlinear causality test on first differences among the 12 regional house prices and the UK as a whole.

$\nrightarrow$ denotes the null of non Granger causality hypothesis (DP). Lag length equals 3 . The $p$-values with *, **, *** represent the significance at $10 \%, 5 \%$ and $1 \%$ level respectively. 
Table 4. Nonlinear (DP) causality (long-run, levels T\&Y)

\begin{tabular}{|c|c|c|c|c|c|c|c|c|c|c|c|c|c|}
\hline LONDONA & $>$ lond & $>$ uk & >north & > york & > swest & $>$ seast & > eanglia & >emidl & $>$ nwest & $>$ wmidl & $>$ wales & $>s \cot$ & >irel \\
\hline p-values & & $0.05679^{*}$ & 0.10897 & 0.13031 & $0.07274^{*}$ & $0.05397^{*}$ & $0.07602^{*}$ & $0.07427^{*}$ & 0.10733 & $0.07148^{*}$ & $0.08143^{*}$ & $0.09216^{*}$ & 0.21291 \\
\hline UKA & $>$ lond & $>$ uk & $>$ north & $>$ york & > swest & $>$ seast & > eanglia & >emidl & $>$ nwest & $>$ wmidl & $>$ wales & $>s \cot$ & >irel \\
\hline p-values & 0.83058 & & 0.10202 & 0.11870 & 0.81046 & 0.81172 & 0.53298 & $0.07425^{*}$ & $0.09286^{*}$ & $0.06975^{*}$ & $0.06641^{*}$ & $0.08800^{*}$ & $0.06723^{*}$ \\
\hline NORTHA & $>$ lond & $>$ uk & $>$ north & $>$ york & > swest & $>$ seast & > eanglia & >emidl & $>$ nwest & $>$ wmidl & $>$ wales & $>s \cot$ & >irel \\
\hline$p$-values & 0.51275 & 0.71880 & & 0.19515 & 0.26771 & 0.21809 & 0.21874 & 0.78727 & 0.16567 & 0.77749 & 0.50000 & 0.11614 & $0.072^{*}$ \\
\hline YORKA & $>$ lond & $>$ uk & $>$ north & $>$ york & >swest & $>$ seast & > eanglia & >emidl & $>$ nwest & $>$ wmidl & $>$ wales & $>s \cot$ & >irel \\
\hline p-values & 0.50181 & 0.89866 & $0.09204^{*}$ & & 0.27544 & 0.26902 & 0.21702 & 0.51597 & $0.09952^{*}$ & 0.65353 & 0.41429 & $0.09256^{*}$ & $0.06685^{*}$ \\
\hline SOUTH WESTA & $>$ lond & $>$ uk & $>$ north & $>$ york & $>$ swest & $>$ seast & > eanglia & >emidl & >nwest & $>$ wmidl & $>$ wales & $>s \cot$ & >irel \\
\hline p-values & 0.62238 & $0.05348^{*}$ & 0.10635 & 0.11460 & & 0.59521 & 0.10292 & $0.06084^{*}$ & $0.09730^{*}$ & $0.05771^{*}$ & $0.06299^{*}$ & $0.09278 *$ & $0.01597^{* *}$ \\
\hline SOUTHEASTA & $>$ lond & $>$ uk & $>$ north & $>$ york & > swest & $>$ seast & > eanglia & >emidl & $>$ nwest & $>$ wmidl & $>$ wales & $>s \cot$ & >irel \\
\hline p-values & 0.24869 & $0.04969 * *$ & 0.10602 & 0.12437 & $0.05020^{*}$ & & $0.07045^{*}$ & $0.06976^{*}$ & 0.10035 & $0.06694^{*}$ & $0.07599^{*}$ & $0.09319^{*}$ & $0.04840^{* *}$ \\
\hline EAST ANGLIAA & $>$ lond & $>$ uk & $>$ north & $>$ york & > swest & $>$ seast & > eanglia & >emidl & $>$ nwest & $>$ wmidl & $>$ wales & $>s \cot$ & >irel \\
\hline p-values & 0.34054 & $0.05554^{*}$ & 0.11273 & 0.12079 & 0.11951 & 0.20061 & & $0.06223^{*}$ & 0.10114 & $0.05738^{*}$ & $0.06464^{*}$ & $0.09702^{*}$ & $0.04726^{* *}$ \\
\hline EAST MIDLAND $\nrightarrow$ & $>$ lond & $>$ uk & $>$ north & $>$ york & > swest & $>$ seast & > eanglia & >emidl & $>$ nwest & $>$ wmidl & $>$ wales & $>\operatorname{scot}$ & >irel \\
\hline p-values & 0.80244 & 0.19406 & 0.10596 & 0.11145 & 0.83839 & 0.62690 & 0.19817 & & $0.09131^{*}$ & $0.07009^{*}$ & $0.06852^{*}$ & $0.08907^{*}$ & 0.11061 \\
\hline NORTH WESTA & $>$ lond & $>$ uk & $>$ north & $>$ york & $>$ swest & $>$ seast & > eanglia & >emidl & $>$ nwest & $>$ wmidl & $>$ wales & $>s \cot$ & >irel \\
\hline p-values & 0.47399 & 0.61662 & $0.08996 *$ & 0.13528 & 0.24963 & 0.25873 & 0.21250 & 0.68275 & & 0.68073 & 0.35864 & $0.08773^{*}$ & $0.07318^{*}$ \\
\hline WEST MIDLANDA & $>$ lond & $>$ uk & $>$ north & $>$ york & > swest & $>$ seast & > eanglia & >emidl & >nwest & $>$ wmidl & $>$ wales & $>s \cot$ & >irel \\
\hline p-values & 0.56636 & 0.47194 & 0.10619 & 0.11384 & 0.22488 & 0.24145 & 0.19916 & 0.12412 & $0.09398^{*}$ & & $0.05757^{*}$ & $0.09074^{*}$ & 0.11293 \\
\hline WALESA & $>$ lond & $>$ uk & $>$ north & $>$ york & > swest & $>$ seast & > eanglia & >emidl & $>$ nwest & $>$ wmidl & $>$ wales & $>s \cot$ & >irel \\
\hline p-values & 0.52065 & 0.88305 & $0.09466^{*}$ & 0.12037 & 0.23331 & 0.24362 & 0.20065 & 0.36653 & $0.09648^{*}$ & 0.38299 & & $0.08738^{*}$ & 0.10496 \\
\hline SCOTLAND-A & $>$ lond & $>$ uk & $>$ north & $>$ york & > swest & $>$ seast & > eanglia & $>$ emidl & $>$ nwest & $>$ wmidl & $>$ wales & $>s \cot$ & >irel \\
\hline p-values & 0.43415 & 0.88674 & 0.24765 & 0.33115 & 0.20517 & 0.21650 & 0.22509 & 0.63675 & 0.15201 & 0.48938 & 0.44246 & & $0.05690^{*}$ \\
\hline IRELANDA & $>$ lond & $>$ uk & $>$ north & $>$ york & $>$ swest & $>$ seast & > eanglia & >emidl & $>$ nwest & $>$ wmidl & $>$ wales & $>s \cot$ & >irel \\
\hline p-values & 0.11165 & $0.09518^{*}$ & 0.13340 & 0.17356 & 0.15144 & $0.09821^{*}$ & 0.10184 & 0.10771 & 0.14135 & 0.10227 & 0.14821 & $0.08900^{*}$ & \\
\hline
\end{tabular}

Notes: This table reports the results of nonlinear causality test on levels among the 12 regional house prices and the UK as a whole.

$\nrightarrow$ denotes the null of non Granger causality hypothesis (DP). Lag length equals 3. The $p$-values with *,**, *** represent the significance at $10 \%, 5 \%$ and $1 \%$ level respectively. 
Table 4 presents the case where we allow the long-run (levels) causality to be nonlinear. This would allow us to take into account potential co-integrating relationships. The more significant pairs are observed in London (8 out of 12), South West (7 out of 12) and South East (7/12). These results highlight the importance of the housing market in London for the UK as the region with overall the more significant pairs. Table 5 summarise the results. Note that linear causality is based on $F$-tests from a VAR framework whereas the nonlinear analysis from a pairwise approach of the DP. For the linear case (the first two columns of results) we cannot identify any region with consistently high number of significant predictability. When we consider the nonlinear case, London emerges as the most significant region in the UK with 10(8) significant pairs in the short-run (long-run).

Table 5. Significant results for each region

\begin{tabular}{lcccc}
\hline \hline Regions & $\begin{array}{c}\text { Linear } \\
\text { short-run } \\
\text { causality }\end{array}$ & $\begin{array}{c}\text { Linear long-run } \\
\text { causality(T\&Y) }\end{array}$ & $\begin{array}{c}\text { Nonlinear short-run } \\
\text { causality (DP) }\end{array}$ & $\begin{array}{c}\text { Nonlinear long-run } \\
\text { causality (DP) }\end{array}$ \\
\cline { 2 - 5 } & Significant & Significant & $\begin{array}{c}\text { Significant } \\
\text { pairs }\end{array}$ & $\begin{array}{c}\text { Significant } \\
\text { pairs }\end{array}$ \\
\hline \hline UK & & & $9 / 12$ & $6 / 12$ \\
LONDON & $3 / 11$ & $4 / 11$ & $10 / 12$ & $8 / 12$ \\
NORTH & $2 / 11$ & $7 / 11$ & $7 / 12$ & $1 / 12$ \\
YORK & $3 / 11$ & $7 / 11$ & $6 / 12$ & $4 / 12$ \\
SOUTH WEST & $3 / 11$ & $10 / 11$ & $8 / 12$ & $7 / 12$ \\
SOUTH EAST & $5 / 11$ & $8 / 11$ & $8 / 12$ & $7 / 12$ \\
EAST ANGLIA & $4 / 11$ & $7 / 11$ & $8 / 12$ & $6 / 12$ \\
EAST MIDLANDS & $5 / 11$ & $8 / 11$ & $6 / 12$ & $4 / 12$ \\
NORTH WEST & $4 / 11$ & $5 / 11$ & $9 / 12$ & $3 / 12$ \\
WEST MIDLANDS & $1 / 11$ & $9 / 11$ & $4 / 12$ & $3 / 12$ \\
WALES & $3 / 11$ & $8 / 11$ & $11 / 12$ & $3 / 12$ \\
SCOTLAND & $5 / 11$ & $8 / 11$ & $3 / 12$ & $1 / 12$ \\
N IRELAND & $4 / 11$ & $6 / 11$ & $6 / 12$ & $3 / 12$ \\
\hline \hline
\end{tabular}

Notes: Linear causality is based on $F$-tests from a VAR framework and DP stands for the procedure proposed by Diks and Panchenko (2006).

\section{Conclusions}

The aim of this paper is to examine both the short-run and the long-run predictability among the UK regional housing market. We employ data that span from 1983:Q1 to 2012:Q4 for 12 UK regions (plus the aggregate UK index). Within a VAR framework we examine linear causality in the short-run and the long-run. We then relax the assumption of linearity and employ the nonparametric causality test approach of Diks and Panchenko (2006). Our basic conclusions are (i) long-run causality among UK regional housing market is mainly linear, (ii) short-run predictability in the UK regional housing market is non-linear and (iii) London's effect on the rest of the UK housing market is the most important and is mainly short-run and nonlinear. 
Acknowledgements. We would like to thank Matias Mayor and an anonymous referee for useful comments.

\section{References}

Alagidede, P., Panagiotidis, T. and Zhang, X. (2011) Causal relationship between stock prices and exchange rates, The Journal of International Trade \& Economic Development, 20(1), 67-86.

Ashworth, J. and Parker, S. (1997) Modelling regional house prices in the UK, Scottish Journal of Political Economy, 44(3), 225-246.

Bampinas, G. and Panagiotidis, T. (2014) On the relationship between oil and gold before and after financial crisis: linear, nonlinear and time-varying causality testing, mimeo.

Brooks, C. (2014) Introductory econometrics for finance, Cambridge University Press.

Cook, S. (2003) The convergence of regional house prices in the UK, Urban Studies, 40(11), 2285-2294.

Drake, L. (1993) Testing for convergence between UK regional house prices, Regional Studies, 29(4), 357-366.

Diks, C. and Panchenko, V. (2005) A note on the Hiemstra-Jones test for Granger noncaussality, Studies in Nonlinear Dynamics and Econometrics, 9(2), 1-9.

Diks, C. and Panchenko, V. (2006) A new statistic and practical guidelines for nonparametric Granger causality testing, Journal of Economic Dynamics \& Control, 30(9-10), 1647-1669.

Engle, R. and Granger, C. (1987) Co-integration and error correction: representation, estimation and testing, Econometrica, 55(2), 251-276.

Granger, C.W.J. (1969) Investigating causal relations by econometric models and cross-spectral methods, Econometrica, 37(3), 424-438.

Holmes, M.J. and Grimes, A. (2008) Is there long-run convergence among regional house prices in the UK?, Urban Studies, 45(8), 1531-1580.

Holmes M.J., Otero, J. and Panagiotidis, T. (2011) Investigating regional house price convergence in the United States: evidence from a pair-wise approach, Economic Modelling, 28(6), 2369-2376.

Hiemstra, C. and Jones, J.D. (1994) Testing for linear and nonlinear Granger causality in the stock price-volume relation, Journal of Finance, 49(5), 1639-1664.

Im, K.S., Pesaran, M.H. and Shin, Y. (2003) Testing for unit roots in heterogeneous panels, Journal of Econometrics, 115(1), 53-74.

Johansen, S. (1995) Likelihood-based inference in cointegrated vector autoregressive model, Oxford University Press, Oxford.

Kuethe, T.H. and Pede, V.O. (2011) Regional housing price cycles: a spatio-temporal analysis using US state-level data, Regional Studies, 45(5), 563-574.

Macdonald, R. and Taylor, M. (1993) Regional house prices in Britain: long-run relationships and short-run dynamics, Scottish Journal of Political Economy, 40(1), 43-55.

Toda, H.Y. and Yamamoto, T. (1995) Statistical inference in vector autoregressions with possibly integrated processes, Journal of Econometrics, 66(1-2), 225-250.

Tsai, I-C. (2014) Ripple effect in house prices and trading volume in the UK housing market: new viewpoint and evidence, Economic Modelling, 40, 68-75. 